\title{
Plantes utilisées pour attirer les essaims de l'abeille domestique (Apis mellifera adansonii Latreille) au Burkina Faso
}

\author{
Issa NOMBRE ${ }^{1,2^{*}}$, Paul SCHWEITZER ${ }^{3}$, Joseph ISSAKA BOUSSIM ${ }^{2}$ et \\ Jeanne MILLOGO/RASOLODIMBY ${ }^{2}$ \\ ${ }^{1}$ Institut des Sciences, 01 BP 1757 Ouagadougou 01, Burkina Faso. \\ ${ }^{2}$ Laboratoire de Biologie et Ecologie Végétales, Université de Ouagadougou, \\ 03 BP 7021 Ouagadougou 03, Burkina Faso. \\ ${ }^{3}$ Laboratoire d'Analyses et d'Ecologie Apicole, Centre d'Etudes Techniques Apicole de Moselle, \\ Lorraine, 1A, Rue Jean-Baptiste de la Salle, 57310 Guenange, France. \\ *Auteur correspondant, E-mail : nombre_issa@yahoo.fr ; Tel : (00226) 70701960
}

\section{RESUME}

Les pratiques apicoles modernes utilisées pour attirer les essaims de l'abeille domestique dans les ruches engendrent des coûts élevés à cause du matériel qui est souvent importé. Or au niveau des pratiques traditionnelles, il existe un ensemble de plantes qui sont utilisées depuis des temps lointains pour attirer les essaims d'abeilles dans les ruches traditionnelles. Une enquête "ethnoapicole" menée dans deux zones phytogéographiques du Burkina Faso a permis de recenser treize espèces végétales reparties en douze genres et huit familles botaniques qui sont utilisées par les apiculteurs traditionnels pour attirer les essaims d'Apis mellifera adansonii Latreille. Un meilleur conditionnement de ces plantes permettrait de les utiliser avec les ruches modernes pour leur colonisation, constituant ainsi une alternative à l'importation du matériel de capture et/ou d'attraction des essaims, une réduction des frais d'installation des ruchers pour un développement et une extension de l'apiculture moderne dans les zones rurales des pays africains.

(C) 2009 International Formulae Group. All rights reserved.

Mots clés : plante, "ethnoapicole", apiculture, essaim, Burkina Faso.

\section{INTRODUCTION}

L'apiculture est une pratique séculaire au Burkina Faso comme en témoigne la présence de nombreuses familles d'apiculteurs traditionnels. Plusieurs techniques apicoles et/ou matériels sont utilisées pour exploiter plus ou moins efficacement les colonies d'abeilles (Villières, 1987; Nombré et al., 2002). Si certaines techniques telles que l'utilisation du feu pour récolter le miel ou pour l'extraire, le pressage des rayons de miel à la main pour extraire le miel peuvent altérer la qualité de ce dernier (Carroll 1997), l'utilisation des organes de plantes pour enfumer les ruches afin d'attirer les essaims sauvages de l'abeille domestique constitue la manière la plus facile pour obtenir de nouvelles colonies (Paterson, 2008). En effet, les plantes sont disponibles et accessibles d'une part et d'autre part, elles pourraient remplacer les charmes abeilles ou les parfums d'Aristée généralement importés d'Europe et nécessitant des coûts supplémentaires. Or le développement des activités apicoles peut constituer des alternatives à la lutte contre la paupérisation du monde rural car l'apiculture bien conduite est une source de revenues (Krell, 1996 ; Bradbear, 2005). De plus, les abeilles sont les principaux insectes pollinisateurs jouant ainsi un rôle dans la conservation de la phytodiversité et constituant des bio indicateurs de la pollution de l'environnement (Ponikvar et al., 2005; Vaissière et al., 2005). 
C'est pour connaître les plantes utilisées pour attirer dans les ruches les essaims de l'abeille domestique que la présente étude a été entreprise dans deux zones phytogéographiques du Burkina Faso que sont Nazinga dans le secteur phytogéographique sud soudanien et Garango dans le secteur nord soudanien. Elle vise à recenser ces plantes à l'aide des enquêtes "ethnoapicoles" menées auprès des apiculteurs traditionnels de ces deux zones.

\section{MATERIEL ET METHODES Milieux d'étude}

Les enquêtes ont été menées dans la zone de Garango située dans le secteur phytogéographique nord soudanien entre $11^{\circ} 47$ de latitude Nord et $0^{\circ} 33$ longitude Ouest et dans celle de Nazinga dans le secteur phytogéographique sud soudanien (Fontès et Guinko, 1995) entre $11^{\circ} 03$ de latitude nord et $1^{\circ} 19$ de longitude Ouest (Figure 1). Les précipitations annuelles varient de $750 \mathrm{~mm}$ à $1000 \mathrm{~mm}$ dans la zone de Garango et de 800 mm à 1100 mm dans celle de Nazinga. Dans la zone de Garango, la végétation est une savane arbustive à graminées annuelles présentant partout l'allure de paysage agreste dominé par quelques espèces utiles telles que Vitellaria paradoxa Gaertn (Sapotaceae), Parkia biglobosa (Jacq.) Benth (Mimosaceae), Lannea microcarpa Engl. et $\mathrm{Kr}$. (Anacardiaceae), Adansonia digitata L. (Bombacaceae), Tamarindus indica L. (Caesalpiniaceae), Acacia dudgeoni Craib. ex Holl. (Mimosaceae). Les bois sacrés à Anogeissus leiocarpus (DC.) G. et Perr. (Combretaceae) sont souvent prédominants. La végétation de la zone de Nazinga est une savane herbeuse à arborée avec des forêts galeries le long des principaux cours d'eau que sont la Sissili et ses affluents dont le Nazinga et le Dawévelé.

\section{Methodes}

L'étude repose sur des enquêtes de type semi structurées conduites en fin de saison sèche et fraîche (période qui correspond à la préparation des ruches traditionnelles par les apiculteurs en vue de la capture des essaims). Les personnes interrogées sont des apiculteurs traditionnels possédant au moins une ruche traditionnelle installée et colonisée. L'enquête a été réalisée dans 10 villages de la zone de
Nazinga et dans 13 villages de la zone de Garango.

Les noms des plantes sont transcrits en langue vernaculaire locale par des enquêteurs originaires des deux zones. A l'aide d'un échantillon fertile de la plante, nous déterminons son nom scientifique à l'aide de l'herbier du Laboratoire de Biologie et Ecologie Végétale de l'Université de Ouagadougou et de la flore Hutchinson.

\section{RESULTATS ET DISCUSSION}

Un total de 103 apiculteurs traditionnels d'âge compris entre 33 et 55 ans ont été enquêtés.

Les plantes retenues sont celles qui ont été citées par zone par au moins dix apiculteurs traditionnels.

Les organes des différentes plantes utilisées pour attirer les essaims d'Apis mellifera adansonii Lat. sont recensés dans le tableau 1.

Treize espèces végétales appartenant à douze genres et huit familles botaniques sont utilisées seules ou en association pour enfumer les ruches afin d'attirer efficacement les essaims de l'abeille domestique.

La technique d'enfumage consiste à creuser dans le sol un trou de diamètre égal à celui de l'ouverture de la ruche. Dans ce trou, les différents organes préalablement séchés y sont déposés en association avec de la bouse de vache sèche qui sert de combustibles. L'ensemble est allumé et le trou est recouvert avec la ruche dont l'intérieur est préalablement crépi avec de l'argile ou de la bouse de vaches pétrie dans de l'eau. Ce crépissage a pour objectif de protéger les abeilles de la lumière, des effets des variations de la température, des infiltrations des eaux de pluie. Il permet également une meilleure rétention des essences contenues dans la fumée lors de l'enfumage (Nombré, 2003). Lorsque la combustion est terminée, la ruche est retirée et laissée au repos pour qu'elle se refroidisse. Elle est ensuite déposée dans un arbre ou dans un bosquet à même le sol pour attirer les essaims. Ces ruches, une fois colonisées, les abeilles vont propoliser leur intérieur et les rendre aseptiques. Pour Paterson (2008), ces organes peuvent être frottés directement à l'intérieur de la ruche ou attachés à l'extérieur. 


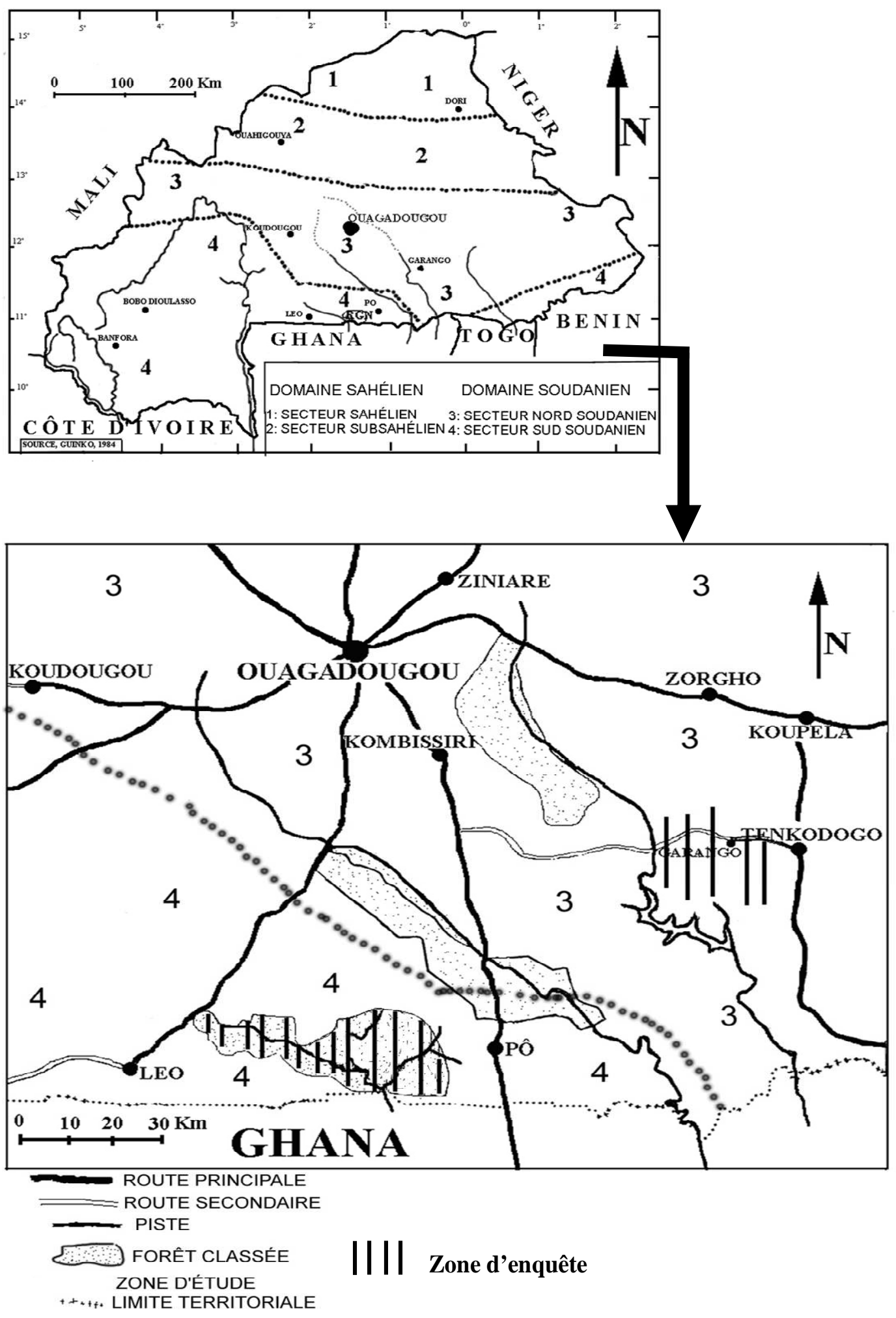

Figure 1: Localisation des sites d'études. 
Tableau 1: Organes des plantes utilisés pour attirer les essaims sauvages d'Apis mellifera adansonii Lat. dans les zones de Garango et de Nazinga.

\begin{tabular}{|c|c|c|c|c|}
\hline Familles & Espèces végétales & Noms vernaculaires & Organes utilisés & Zones \\
\hline \multirow{2}{*}{ Caesalpiniaceae } & $\begin{array}{l}\text { Piliostigma reticulatum } \\
\text { (DC.) Hoscht }\end{array}$ & Semellier & Fruits secs & G. \\
\hline & $\begin{array}{l}\text { Piliostigma thonningii } \\
\text { (Schumach.) Milne-Redh }\end{array}$ & Semellier & Fruits secs & N. G. \\
\hline \multirow{2}{*}{ Combretaceae } & $\begin{array}{l}\text { Combretum glutinosum } \\
\text { Perr. ex DC. }\end{array}$ & Chigommier & Tiges feuillées & N. G. \\
\hline & $\begin{array}{l}\text { Guiera senegalensis J. F. } \\
\text { Gmel. }\end{array}$ & Guiera du Sénégal & Tiges feuillées & N. G. \\
\hline \multirow[t]{2}{*}{ Ebenaceae } & $\begin{array}{l}\text { Diospyros mespiliformis } \\
\text { Hochst. ex A. Rich }\end{array}$ & $\begin{array}{l}\text { Ebénier de l'Ouest } \\
\text { africain }\end{array}$ & $\begin{array}{l}\text { Jeunes feuilles, } \\
\text { fruits secs }\end{array}$ & G. \\
\hline & Hyptis spicigera Lam. & & Tiges feuillées & G. \\
\hline \multirow[t]{3}{*}{ Lamiaceae } & $\begin{array}{l}\text { Leucas martinicensis } \\
\text { (Jacq.) R. Br. }\end{array}$ & & Tiges feuillées & G. \\
\hline & Ocimum basilicum $\mathrm{L}$. & Basilic & Tiges feuillées & N. G. \\
\hline & Acacia seyal Del. & Mimosa épineux & Fruits secs, & G. \\
\hline Mimosaceae & Parkia biglobosa (Jacq.) & Néré & $\begin{array}{l}\text { Décocté des graines } \\
\text { bouillies }\end{array}$ & N. \\
\hline Poaceae & $\begin{array}{l}\text { Cymbopogon schoenanthus } \\
\text { subsp. Proximus }\end{array}$ & & $\begin{array}{l}\text { Inflorescences } \\
\text { sèches }\end{array}$ & G. \\
\hline Polygalaceae & $\begin{array}{l}\text { Securidaca } \\
\text { Longepedunculata Fres. }\end{array}$ & Arbre à serpent & Racines & N. \\
\hline Sapotaceae & Vitellaria paradoxa Gaertn & Karité & Ecorces & N. \\
\hline
\end{tabular}

Piliostigma reticulatum (DC.) Hochst et $P$. thonningii (Sch.) Miln.-Redh. sont des arbustes typiquement soudaniens à nombreux rejets, mesurant 0,5 à $2 \mathrm{~m}$ de haut, à feuilles bilobées, à fleurs blanches unisexuées et dont les fruits sont des gousses. Le premier a une aire géographique de répartition qui se limite au secteur nord soudanien. Le genre Piliostigma est aussi signalé par Arbonnier (2000) comme plante intervenant dans l'enfumage des ruches.

Hyptis spicigera Lam., Leucas martinicensis (Jacq.) Ait., Ocimum americanum L. sont des plantes herbacées annuelles ou semi annuelles vivant souvent dans des zones inondables, à tiges à section quadrangulaire, finement pubescentes. Ce sont des plantes aromatiques à l'instar de Cymbopogon schoenanthus Spreng. (Poaceae) dont les extrémités fleuries sont très parfumées (Millogo-Rasolodimby et al., 1998) qui est une plante herbacée pluriannuelle. Le genre Ocimum a été signalé par Paterson (2008) comme attire essaim utilisé par les apiculteurs au Kenya. Guiera senegalensis J.F. Gmel. et Combretum glutinosum Perr. sont des arbustes buissonnants mesurant $1 \mathrm{~m}$ de haut, à 
inflorescences en épis axillaires ou en glomérules axillaires avec des fleurs jaunes verdâtres à blanchâtres. Les fruits sont linéaires ou ailés.

Securidaca longepedunculata Fres., est un arbuste de 3 à $5 \mathrm{~m}$ de haut, à fleurs pourpres et fruits ailés. Elle est reconnue comme répulsive des serpents (Arbonnier, 2002) et pourrait dans ce cas être utilisée pour protéger les ruches contre ces reptiles.

\section{Conclusion}

Des organes végétatifs et reproducteurs de plusieurs plantes sont utilisés pour enfumer les ruches afin d'attirer les essaims sauvages de l'abeille domestique dans les deux zones. Certains organes de plantes pourraient être réduits en poudre et utilisés dans la préparation des ruches modernes pour attirer les essaims; cela permettra également de remplacer le matériel de capture des essaims généralement importés. Cela permettra de réduire le coût de la modernisation de l'apiculture traditionnelle.

\section{REFERENCES}

Arbonnier M. 2000. Arbres, Arbustes et Lianes des Zones Sèches d'Afrique de l'Ouest. CIRAD-MNHN-UICN; 541p.

Bradbear N. 2005. Apiculture et moyens d'existence durable. FAO Brochure sur la Diversification, 160p.

Carroll MSCT. 1997. Beekeeping, a beginner's guide. Association for Better Land Husbandry, $37 \mathrm{P}$.
Fontès J, Guinko S. 1995. Carte et notice de la végétation et de l'occupation du sol au Burkina Faso. UMR-ICIV, 9964 du CNRS/Univ. Paul Sabatier, Toulouse, IDR/FAST, IRBET/CNRST et MET, Ouagadougou, 78p.

Krell R. 1996. Value-added products from beekeeping. FAO Agricultural Services Bulletin $\mathrm{N}^{\circ} 124,395 \mathrm{p}$.

Millogo-Rasolodimby J, Nacoulma O, Samaté AD. 1998. Les utilisations des poaceae aromatiques au Burkina Faso. Rev. Med. Pharm. Afr., 11(12): 157-164.

Nombré I, Sawadogo M, Boussim IJ, Guinko S. 2002. Beekeeping in Burkina Faso. Bees for Development Journal, 65: 9-9.

Nombré I. 2003. Etude des potentialités mellifères de deux zones du Burkina Faso Garango (Province du Boulgou) et Nazinga (Province du Nahouri). Thèse de Doct. Unique, Univ. de Ouagadougou, Burkina Faso, 169p.

Paterson PD. 2008. L'Apiculture. Coéditions Quae-CTA-Presses Agronomiques de Gembloux; 160p.

Ponikvar M, Snajder J, Sedej B. 2005. Honey as a bioindicator for environmental pollution with $\mathrm{SO}_{2}$. Apidologie, 36: 403409.

Vaissière B, Morison N, Carré G. 2005. Abeilles pollinisation et biodiversité. Abeille et Compagnie, 106 : 10-14.

Villières B. 1987. L'apiculture en Afrique Tropicale. Le point sur; dossier 11; GRET, ACCT, AFVP, 220p. 